\title{
Color Doppler Ultrasound in the pre-histological determination of the biological character of major salivary gland tumors
}

\author{
Pavel Strympl ${ }^{a}$, Michal Kodaj ${ }^{\mathrm{b}}$, Tomas Bakaj ${ }^{\text {, }}$ Pavel Kominek ${ }^{\mathrm{a}}$, Ivo Starek ${ }^{c}$, Ivan Sisola ${ }^{\mathrm{d}}$, Hana Tomaskova ${ }^{\mathrm{e}}$, Petr Matousek ${ }^{\mathrm{a}}$
}

\begin{abstract}
Objectives. The aim of the study was to assess the use of color Doppler ultrasound in the pre-histological determination of the biological features of salivary gland tumors.

Material and Methods. Ninety-six patients with major salivary gland tumors of unknown histology were examined and operated on in our clinics. They were pre-operatively examined using ultrasound imaging with color Doppler. Peak systolic velocity (PSV) was measured and pulsatility index (PI) and resistive index (RI) were calculated on the pulsed wave traces. The Doppler flow parameters were correlated with clinical stage and tumour type (benign/carcinoma) as confirmed by final histological diagnosis. For the correlations, the tumors were categorized into 3: benign (group I), malignant stages I+II (group II), malignant stages III+IV (group III).

Results. The average PSV value was $22.15 \mathrm{~cm} / \mathrm{s}$ for benign and $32.74 \mathrm{~cm} / \mathrm{s}$ for all malignant tumors. The average RI value was 0.77 for benign and 0.86 for all malignant tumors. The average PI value for benign tumors was 2.85 and 3.14 for all malignant tumors. No significant differences between benign and malignant tumors in terms of $S V$ and PI values were found. The RI values for benign tumors differed significantly from those of malignant ones $(P=0.021)$. There were no significant differences in average PSV, PI and RI values in relation to salivary gland tumor group - I, II, III. There was no confirmation of the reported applicability of PSV and PI values in differenting benign from malignant tumors.

Conclusion. We were not able to demonstrate significant differences in Doppler flow parameters PSV and PI between benign tumors and carcinomas. Only the RI could be used to differentiate them. There were also no significant differences in PSV, PI and RI values between low (I+II) and high (III+IV) clinical tumour stage.
\end{abstract}

Key words: color Doppler ultrasound, salivary gland, tumor

Received: April 23, 2012; Accepted: July 10, 2012; Available online: September 5, 2012

http://dx.doi.org/10.5507/bp.2012.074

${ }^{a}$ Department of Otolaryngology, University Hospital Ostrava, Czech Republic

${ }^{b}$ Department of Radiology, University Hospital Ostrava

'Department of Otorhinolaryngology, Faculty of Medicine and Dentistry, Palacky University Olomouc

${ }^{d}$ Department of Radiology, Faculty of Medicine and Dentistry, Palacky University Olomouc

${ }^{e}$ Department of Epidemiology and Public Health, Faculty of Health Studies, University of Ostrava

Corresponding author: Pavel Strympl, e-mail: pavel.strympl@fno.cz

\section{INTRODUCTION}

Salivary gland neoplasms account for $3 \%$ of all tumors ${ }^{1}$. Most are benign, with the parotid being the most common site of origin. The smaller the involved salivary gland, the higher the probability of malignancy, the rate of which thus increases from $20 \%-25 \%$ in the parotid, through $40 \%-50 \%$ in the submandibular, to $50 \%-81 \%$ in the sublingual and minor salivary glands $\mathrm{s}^{2-5}$.

Relevant important preoperative information is the determination of their biological character. Since sole clinical investigation lacks the necessary reliability in this respect, imaging methods are being applied. Of these and due to its zero invasiveness and cost effectiveness, ultrasound is considered an essential imaging method. Its specificity can be substantially improved by the use of the color Doppler method, mapping the blood flow in tumor supplying vessels. Color Doppler criteria are used to distinguish benign from malignant tumors, particularly in terms of grade of intratumor vascularity, pattern of vascular supply, and flow parameters (Table 1) $\left(\right.$ ref. $\left.^{6}\right)$. Some publications reports on the usefulness of color Doppler
US in the pre-histological determination of the biological character of the tumors ${ }^{3,4,6,7}$ while others do not ${ }^{8,9}$.

The main aim of this study was to test the use of color Doppler US in the preoperative assessment of the biological character of salivary gland tumors which would enable us to match the extent of surgery to their real dignity.

\section{MATERIAL AND METHODS}

A total of 96 patients with parotid gland tumors were examined in this prospective study at our clinics between June 2006 and August 2011. Fifty-four women and 42 men were enrolled, average age 57.3 and 60.4 (mean 58.7) years. Sixty-six $(68 \%)$ tumors were benign and $30(32 \%)$ were malignant (Table 2). Malignant tumors were found in 17 women and 13 men. In 19 patients, the set of tested color Doppler parameters was not complete. Thus, the PSV value was measured in 95 tumors, RI in 94 tumors and PI in 74 tumors.

The color Doppler US was the basic preoperative diagnostic tool. When a deep lobe parotid tumor was suspect- 
Table 1. Color Doppler ultrasound parameters in major salivary gland tumors diagnostics.

\begin{tabular}{l} 
Color Doppler US parameters \\
\hline Quantitative parameters \\
\hline The number of supplying vessels. Low-differentiated ma- \\
lignant tumors usually have richer vessel supply when \\
compared with benign tumors ${ }^{6,7}$. We can see 3 and more \\
tumor supplying arteries in the case of malignant tu- \\
mors $^{2}$.
\end{tabular}

Qualitative parameters

- Peak systolic velocity (PSV). PSV in the arteries supplying benign tumors are usually lower that $25 \mathrm{~cm} / \mathrm{s}$ ( ref. $^{2}$ ). Higher Doppler velocities can be seen in the vessels of malignant tumors. Velocity higher than $60 \mathrm{~cm} / \mathrm{s}$ was found in the malignant tumor vessels. Dock et al. recommends PVS $40 \mathrm{~cm} / \mathrm{sec}$ as the level for differentiation between benign and malignant tumors ${ }^{3}$.

- Pulsatility index (PI). PI is the ratio between PSV and end-diastolic velocity (EDV) difference to the average velocity. The tumor with PI value 1.8 and is considered as potentially malignant ${ }^{7}$.

- Resistive index (RI), so called Pourcelot's index. RI is the ratio between PSV and EDV (end diastolic velocity) difference to PSV. The values 0.8 and higher point to a malignant tumor ${ }^{7}$.

ed or malignancy was confirmed on cytology, magnetic resonance imaging (MRI) or computer tomography (CT) was performed to evaluate the tumor extent and local invasion. The US examination was performed by two independent experienced radiologists. The US linear 6-9 MHz probe was used. The patients were placed in the supine position, with the neck turned to the contralateral side. The region of interest was scanned slowly with a minimal probe pressure.

Longitudinal and transverse scanning planes were used to map the color flow signals within the salivary gland parenchyma. When a tumor vessel was identified, the probe position was adapted into the optimal plane to achieve its ideal display.

The basic US parameters of the tumor (i.e. size, structure and echotexture), adjacent soft tissue invasion and the US signs of potential metastatic lymph node affection were studied. The color mapping of the tumor was then performed with the determination of the count of supplying vessels, in which three flow parameters, namely peak systolic velocity (PSV), resistive index (RI) and pulsatility index (PI) were evaluated. The PSV, RI and PI values equal to or higher than $25 \mathrm{~cm} / \mathrm{s}, 0.8$ and 1.8 , were considered borderlines for malignancy in accordance with the referenced literature (Table 1) $\left(\right.$ ref. $\left.^{2-4,7}\right)$.

The PSV, RI and PI values for benign tumors were compared to those of carcinomas, the differences between two groups were then evaluated with Student t-test.

Individual patients were then categorized according to the biological character and clinical stage of their tumors
Table 2. Histological types of the primary epithelial salivary gland tumors in our study.

\begin{tabular}{lrc}
\hline & $\mathrm{N}^{\circ}$ & $\%$ \\
\hline Benign tumors & 66 & 68.0 \\
pleomorphic adenoma & 37 & 38.1 \\
cystadenolymphoma & 27 & 27.8 \\
bazocellular adenoma & 2 & 2.01 \\
Malignant tumors & 30 & 32.0 \\
squamous cell carcinoma & 6 & 6.3 \\
adenoid cystic carcinoma & 5 & 5.2 \\
mucoepidermoid carcinoma & 4 & 4.2 \\
adenocarcinoma & 4 & 4.2 \\
ductal carcinoma & 4 & 4.2 \\
carcinoma in pleomorphic adenoma & 4 & 4.2 \\
small cell carcinoma & 1 & 1.0 \\
nondifferentiated carcinoma & 1 & 1.0 \\
myoepithelial carcinoma & 1 & 1.0 \\
\hline
\end{tabular}

as follows: benign tumors (group I), malignant tumors staged I+II (group II), malignant tumors staged III+IV (group III). Sixty-six patients (68.75\%) were classified as group I, 17 patients (17.71\%) as group II and 13 patients (13.54\%) as group III.

Peak levels of the PSV, RI and PI in all three groups were compared, using ANOVA. All analyses were performed using the statistical software STATA version 10 . Our results (PSV, PI and RI values) were compared with standard values reported in the literature. The level of significance was $P<0.05$

\section{RESULTS}

\section{The difference in PSV, PI and RI values between benign tumors and carcinomas}

The PSV values ranged from 4 to $90 \mathrm{~cm} / \mathrm{s}$ (mean $25.15 \mathrm{~cm} / \mathrm{s}$ ) for the benign tumor group and from 4 to 68 $\mathrm{cm} / \mathrm{s}$ (mean $32.74 \mathrm{~cm} / \mathrm{s}$ ) for carcinomas. The PI values ranged from 0.64 to 9.4 (mean 2.85) in the benign tumor group and from 0.7 to 13.0 (mean 3.14 ) in the carcinomas. The RI of the benign tumor group ranged from 0.41 to 1.0 (mean 0.77 ) and from 0.2 to 1.2 (mean 0.86 ) in the carcinoma group. A statistically significant difference ( $P=0.021$ ) was found between the benign tumors group and the carcinomas for RI, but not PI and PSV values (Table 3).

\section{The PSV, PI and RI values depending on clinical stage of tumor}

The values of PSV $4-90 \mathrm{~cm} / \mathrm{s}$ (mean $25.15 \mathrm{~cm} / \mathrm{s})$, RI 0.41 - 1.0 (mean 0.77) and PI 0.64 - 9.4 (mean 2.32) were found in group I. The values of PSV, RI, PI in groups II and III were $5-59 \mathrm{~cm} / \mathrm{sec}$ (mean $24.09 \mathrm{~cm} / \mathrm{s}$ ), $0.2-0.9$ (mean 0.83) PI $0.7-13$ (mean 1.92) and $4-68 \mathrm{~cm} / \mathrm{s}$ (mean $34.54 \mathrm{~cm} / \mathrm{s}$ ), $0.68-1.2$ (mean 0.89), $1.3-7.68$ (mean 3.21 ), respectively. The differences between the 
Table 3. The difference of the peak systolic velocity, pulsatility index and resistive index values between benign and carcinomas (B - benign tumor, C - carcinoma, SD - standard deviation).

\begin{tabular}{cccccccc}
\hline Index & Tumor & $\mathrm{N}^{\circ}$ & From & To & Mean & SD & $P$ value \\
\hline \multirow{2}{*}{ PSV } & B & 66 & 4.0 & 90.0 & 25.15 & 17.49 & 0.179 \\
& C & 29 & 4.0 & 68.0 & 32.74 & 27.50 & \\
\multirow{2}{*}{ RI } & B & 66 & 0.41 & 1.0 & 0.77 & 0.16 & 0.021 \\
& C & 28 & 0.2 & 1.2 & 0.86 & 0.19 & \\
PI & B & 51 & 0.64 & 9.4 & 2.85 & 2.32 & 0.642 \\
& C & 21 & 0.7 & 13.0 & 3.14 & 2.76 & \\
\hline
\end{tabular}

Table 4. The peak systolic velocity, pulsatility index and resistive index values depending on the clinical stage of the tumor (I, II, III - groups according to tumor clinical stage, SD - standard deviation, ANOVA - test of variance).

\begin{tabular}{|c|c|c|c|c|c|}
\hline Index & Groups & $\mathrm{N}^{\circ}$ & Mean & SD & $\begin{array}{l}\text { ANOVA } \\
P \text { value }\end{array}$ \\
\hline \multirow{3}{*}{ age } & I & 66 & 54.51 & 15.82 & \multirow{3}{*}{$<0.001$} \\
\hline & II & 17 & 63.47 & 14.48 & \\
\hline & III & 13 & 72.36 & 11.72 & \\
\hline \multirow{3}{*}{ PSV } & I & 66 & 25.15 & 17.49 & \multirow{3}{*}{0.185} \\
\hline & II & 17 & 24.09 & 13.45 & \\
\hline & III & 13 & 34.54 & 21.65 & \\
\hline \multirow{3}{*}{ RI } & I & 66 & 0.77 & 0.16 & \multirow{3}{*}{0.072} \\
\hline & II & 17 & 0.83 & 0.22 & \\
\hline & III & 13 & 0.89 & 0.16 & \\
\hline \multirow{3}{*}{ PI } & I & 51 & 2.85 & 2.32 & \multirow{3}{*}{0.305} \\
\hline & II & 12 & 1.92 & 1.14 & \\
\hline & III & 10 & 3.21 & 1.88 & \\
\hline
\end{tabular}

Table 5. Sensitivity and specificity calculation for parameter RI (B - benign tumor, C - carcinoma, T (-) - negative results, $\mathrm{T}(+)$ - positive results, $\mathrm{RN}$ - right negative result, $\mathrm{FP}$ - false positive result, FN - false negative result, $\mathrm{RP}$ right positive result).

\begin{tabular}{cccc}
\hline & B & C & Total \\
\hline $\mathrm{T}(-)$ & $36(\mathrm{RN})$ & $8(\mathrm{FN})$ & 44 \\
$\mathrm{~T}(+)$ & $32(\mathrm{FP})$ & $20(\mathrm{RP})$ & 52 \\
Total & 68 & 28 & 96 \\
& $\mathrm{RI}$ & \\
\hline
\end{tabular}

PSV, PI and RI values in groups I, II, III were not statistically significant (Table 4).

\section{Using the PSV, PI, RI values reported in the literature for distinguishing benign tumors and carcinomas}

Our study confirmed the use of an RI critical value of 0.8 appearing in the literature ${ }^{2,3,4,7}$ for distinguishing benign tumors and carcinomas. Thirty-six patients had true negative results, in the group of 32 patients false positive results were described, 8 patients had false negative results and 20 patients true positive results. The sensitivity and specificity of the RI parameter in differentiating carcinomas from benign tumors were thus $71.4 \%$ and $52.9 \%$, respectively (Table 5).

No applicability of the PSV value of $25 \mathrm{~cm} / \mathrm{s}$ and PI value 1.8 for differentiation was demonstrated.

The PI parameter differentiated carcinomas from benign tumors with a sensitivity of $61.9 \%$ and a specifity of $49.1 \%$. True negative results were obtained in 26 pa- 
Table 6. Sensitivity and specificity calculation for parameter PI

(B - benign tumor, C - carcinoma, T (-) - negative results, T (+) - positive results, RN - right negative result, $\mathrm{FP}$ - false positive result, $\mathrm{FN}$ - false negative result, $\mathrm{RP}$ - right positive result).

\begin{tabular}{cccc}
\hline & B & C & Total \\
\hline T $(-)$ & $26(\mathrm{RN})$ & $8(\mathrm{FN})$ & 34 \\
$\mathrm{~T}(+)$ & $27(\mathrm{FP})$ & $13(\mathrm{RP})$ & 40 \\
Total & 53 & 21 & 74 \\
\end{tabular}

Table 7. Sensitivity and specificity calculation for parameter PSV

(B - benign tumor, C - carcinoma, T (-) - negative results, T (+) - positive results, RN - right negative result, $\mathrm{FP}$ - false positive result, $\mathrm{FN}$ - false negative result, RP - right positive result).

\begin{tabular}{cccc}
\hline & B & C & Total \\
\hline $\mathrm{T}(-)$ & $42(\mathrm{RN})$ & $11(\mathrm{FN})$ & 53 \\
$\mathrm{~T}(+)$ & $25(\mathrm{FP})$ & $17(\mathrm{RP})$ & 42 \\
Total & 67 & 28 & 95 \\
& PSV: sensitivity $60.7 \%$, specificity $62.7 \%$. \\
\hline
\end{tabular}

tients and in 27 patients false positive results. In 8 cases we found false negative results and in 13 patients true positive results. The PI value was measured in 72 tumors (Table 6).

The parameter PSV can distinguish carcinomas from benign tumors with a sensitivity of $60.7 \%$ and a specificity of $62.7 \%$. In the case of 42 patients, true negative results were found and in 25 patients false positive results. The result from 11 patients were false negative and from another 17 true positive (Table 7).

\section{DISCUSSION}

The role of imaging methods in the evaluation of salivary gland tumors is to define their intra- vs. extraglandular position, to detect local extension, invasion and nodal metastases. All this information can be successfully obtained with US, which is a safe, inexpensive and accurate method $^{10}$, serving as a basic guide for further imaging and treatment strategy. US is also expected to provide us with information about the status of salivary gland tumors, greatly varying between, as well as within, particular histopathologic entities. However, in this aspect, the capability of standard US is rather limited.

A question which remains is whether color Doppler US could be helpful in the determination of the biological nature or even the histopathological type of salivary gland tumor.

Izzo et al. ${ }^{7}$ followed a series of 49 patients with major salivary gland tumors who underwent color and power Doppler US examinations preoperatively. In benign tumors the peritumoral and in much lesser extent intratumoral vascularisation as measured by a color power Doppler US device with 7.5-10 MHz linear probe was modest. On the other hand, carcinomas, due to a number of arteriovenous shunts and massive neovascularization, had a non-homogenous pattern with scattered distribution of vessels.

Martinoli et al. ${ }^{6}$ achieved similar results in their series of 62 patients with major salivary gland tumors. They described significantly more extensive blood supply in the group of malignant tumors.

Some authors found higher vascular resistance as a sign of tumor malignancy. Bradley et al. ${ }^{4}$ examined 56 patients with major salivary gland tumors. PSV was measured and PI and RI calculations were performed and particular values were then correlated with the histolopathological diagnosis. The combination of the real time ultrasound parameters RI and PI diagnosed benign disease with a sensitivity of $89.7 \%$ and specificity of $57.1 \%$. The positive predictive value was $93.6 \%$. There were no significant differences in the colour Doppler appearances in terms of vessel type or intratumour distribution which could separate benign from malignant conditions. No malignant lesion showed the PI and RI lower than 1.8 and 0.8 , respectively.

In a study of 49 patients with benign and 13 patients with malignant tumors Martinoli et al. ${ }^{6}$ did not find significant differences between groups in RI values (range from 0.55 to 1.10 in benign and 0.45 to 1.10 in malignant tumors). Contrary to Martinoli, we confirmed a significant difference in RI, but not in PI values between benign and carcinomas.

PSV is another color Doppler parameter used to analyze the flows in tumor supplying vessels. In a study on 130 benign and malignant tumors (44 mammary gland, 43 liver, 43 other organs) Dock et al. detected generally increased (higher than $40 \mathrm{~cm} / \mathrm{s}$ ) PSV values in the latter ${ }^{3}$. Similar results were presented by Stárek who recommend- 
ed PSV $25 \mathrm{~cm} / \mathrm{s}$ as the level, critical for differentiation between benign and malignant tumors ${ }^{2}$. Martinoli suggested that a systolic peak flow of $60 \mathrm{~cm} / \mathrm{s}$ was a threshold for differentiating benign from malignant lesions. As some of the latter were associated with lower PSV, no reliable conclusions could be drawn ${ }^{6}$. Bradley et al. found PSV elevation $(>50 \mathrm{~cm} / \mathrm{s})$ in some carcinomas, but there was no statistical correlation with malignancy in genera ${ }^{4}$. Schick et al. demonstrated that pulsed Doppler US distinguished benign neoplasms from malignant ones with a $72 \%$ sensitivity and $88 \%$ specificity ${ }^{11}$. The highest PSV observed in benign tumors was $19.9 \pm 7.7 \mathrm{~cm} / \mathrm{s}$, compared with $44.4 \pm 47.7 \mathrm{~cm} / \mathrm{s}$ in malignant tumors. The difference between velocities of benign and malignant tumors was statistically significant ${ }^{11}$. The outcomes of our study are not in accord with these results.

Given different values of PSV, PI and RI considered as threshold between benign and malignant tumors ${ }^{4,7,11}$ we, as well as Gritzmann ${ }^{9}$, are skeptical of their practical application. This view was also shared by Bialek in her paper on US use in salivary gland diagnostics ${ }^{8}$.

Our study, in agreement with Bialek ${ }^{8}$ and Gritzmann ${ }^{9}$ but in contrast to the above studies ${ }^{2-4,7}$ failed to demonstrate the applicability of the PSV value of $25 \mathrm{~cm} / \mathrm{s}$ and PI value of 1.8 as the "break points" for the tumor type. The PSV values were higher than $25 \mathrm{~cm} / \mathrm{s}$ in 26 benign (27.4\%) and 17 malignant tumors (17.9\%) (from 95 PSV measurements in total) in our study. The PI values higher than 1.8 were demonstrated in 25 benign (34.7\%) and 13 malignant tumors (18.1\%) (from 72 PI measurements in total).

Our study confirmed that the parameter RI can be used for differentiating benign from malignant tumours. and confirmed an RI critical value of 0.8 for the distinguishing benign tumors from carcinomas. Doppler US parameter RI differentiates carcinomas from benign tumors with a sensitivity of $71.4 \%$ and specificity of $52.9 \%$.

Overall, significant differences in PSV and PI between benign tumors and carcinomas were not confirmed in contrast to some published papers. Similarly, there were no significant differences in the PSV, PI and RI values for particular clinical stages of the latter.

\section{CONCLUSION}

We confirmed the usefulness of the color Doppler RI parameter in differentiating benign salivary gland tumors from malignant ones. For other parameters (PSV and PI) no significant differences between benign tumors and carcinomas were. The study failed to confirm the benefit of the color Doppler US in the differentiation between benign tumors and clinical stages of carcinomas.

\section{ACKNOWLEDGEMENT}

The authors would like to thank Ms. Vanessa DeRhen, Mr. Jiří Hynčica and Mr. Alexander Oulton for their help and corrections to get this paper in its final form.

Results described in this paper were obtained with support of Grant IGA MZ CR NT/13505.

Authorship contributions: PS: literature search; PS, PK, IS: manuscript writing; PS, IS: study design; PS, TB, MK, ISi, PM: data collection; HT, PS: data analysis and interpretation; HT: statistical analysis and figures; PS, PK, IS: final approval.

Conflict of interest statement: None declared.

\section{REFERENCES}

1. Lee YYP, Wong KT, King AD, Ahujab AT. Imaging of salivary gland tumours. European J Radiol 2008;66:419-36.

2. Stárek I. Choroby slinných žláz. Praha: Grada, 2000. p. 37-47.

3. Dock W, Grabenwoger F, Metz V. Tumor Vascularization: Assessment with Duplex Sonography. Radiology 1991;181:241-4.

4. Bradley M, Durham L, Lancer J. The Role of Colour Flow Doppler in the Investigation of the Salivary Gland Tumour. Clinical Radiology 2000;55:759-62.

5. Ariji Y, Kimura Y, Hayashi N. Power Doppler Sonography of Cervical Lymph Nodes in Patients with Head and Neck Cancer. Am J Neuroradiol 1998; 26:303-7.

6. Martinoli C, Delhi E, Solbiati L. Color Doppler sonography salivary glands. Amer J Roentgenol 1994;163:933-41.

7. Izzo L, Sassayanis P, Frati R. The Role of Echo Colour/Power Doppler and Magnetic Resonance in Expansive Parotid Lesions. J Exp Clin Cancer Res 2004;23:585-92.

8. Bialek EJ, Jakubowski W, Zajkowski P. Ultrasonography of salivary glands: Anatomy and Spatial Relationships, Pathologic Conditions and Pitfalls. RadioGraphics 2006; 26:745-63.

9. Gritzmann N, Rettenbacher T, Hollerweger A. Sonography of the salivary glands. Eur Radiol 2003;13:964-75.

10. Alyas F, Lewis K, Williams L, Mooby AB, Wong KT. Diseases of the submandibular gland as demonstrated using high resolution ultrasound. The British Journal of Radiology 2005;78:362-69.

11. Schick S, Steiner E, Gahleitner A. Differentiation of benign and malignant tumors of the parotid gland: value of pulsed Doppler and color Doppler sonography. Eur Radiol 1998;8:1462-7. 